\title{
GEOFISICA MARINA AL OCCIDENTE DEL MAR CARIBE
}

\author{
JOSE ALBERTO VIVAS VELOSO* y LUIS DEL CASTILLO G.**
}

\begin{abstract}
Gravimetric, magnetic, bathymetric and acoustic reflexion data were gathered by the Cruise LEG 2 (USGS-UNAM-SMN-IMP) along the Mexican and part of Central America coasts covering 32 tracks with a total extent of $4450 \mathrm{~km}$. The observed geophysical anomalies were interpreted based on two dimensional crust models.

Against former ideas, it is suggested that the submarine structure know as Cresta Externa is the possible contact between the sialic continental crust and the intermediate under the Caribbean Sea. The acoustic data for the Yucatan Basin shows the existence of horizontal sedimentary layers deposited on a basement with irregular surface. This indicates that after sedimentation the region was not subjected to important tectonic movements. It is believed that the Caribbean region was formed during Mesozoic time as a consequence of fissure and following continental drift between the American and the Afro-European continents.
\end{abstract}

RESUMO O Cruzeiro LEG 2 (USGS-UNAM-SMN-IMP) realizado nas costas do México e parte da América Central coletou dados de gravimetria, magnetismo, reflexão acústica e de batimetria ao longo de $4450 \mathrm{~km}$, repartidos em 32 linhas. As anomalias geofísicas encontradas foram justificadas através de modelos bidimensionais representativos da crosta.

Em contraste aos conceitos anteriores, sugere-se que a estrutura submarina denominada Cresta Externa, seja o possível contato entre a crosta siálica do continente e a intermediária abaixo do Mar do Caribe. A informação acústica na Bacia de Yucatán indica a presença de sedimentos horizontais depositados sobre um embasamento com superficie bastante irregular. Isso parece indicar que, em época posterior à sedimentação, não se apresentaram, na área, movimentos tectônicos de grande magnitude. Acredita-se que a região do Caribe tenha se originado no Mesoź́ico como conseqüuencia da fissura e da subseqüente deriva dos continentes americano e afromeuropeu.

INTRODUCCION Como parte de los programas de la International Decade of Ocean Exploration (IDOE) los Estados Unidos, a través del United States Geological Survey (USGS) iniciaron algunos trabajos de investigación geofisica y geologia marina en las áreas continentales del Golfo de México y Mar Caribe.

Científicos y observadores mexicanos del Instituto de Geofisica de la Universidad Nacional Autónoma de México (UNAM), Instituto Mexicano del Petróleo (IMP) y de la Comisión Oceanográfica (SMN), colaboraron en las operaciones marinas, realizando el Departamento de Exploración del Instituto citado en primer término, las labores de investigación consistentes en el procesamiento y la interpretación de los datos obtenidos durante el crucero.

Ese estudio versa sobre una parte del levantamiento efectuado en el Mar Caribe denominado LEG 2. Su objetivo es el reconocimiento regional de estructuras profundas, las cuales podrían ayudar en la comprensión de la história geológica del Caribe basada en la teoria de la tectónica global de placas.

El área estudiada comprende la región occidental del Mar Caribe, cuyo límite superior es el paralelo $22^{\circ} 50^{\prime} \mathrm{N}$, hacia el este queda limitada por el meridiano $85^{\circ} 05^{\prime} \mathrm{W}$, hacia el oeste por las costas de la Península de Yucatán, abarcando hasta Honduras Británicas (Belice) y hacia el sur por las costas de Guatemala y Honduras (Figura 1).

*Departamento de Geociências, Universidade de Brasilia - 70000, Brasília (DF), Brasil

**Departamento de Exploración - Instituto de Geofisica, Universidad Nacional Autónoma de México - México, 20 (DF), México 


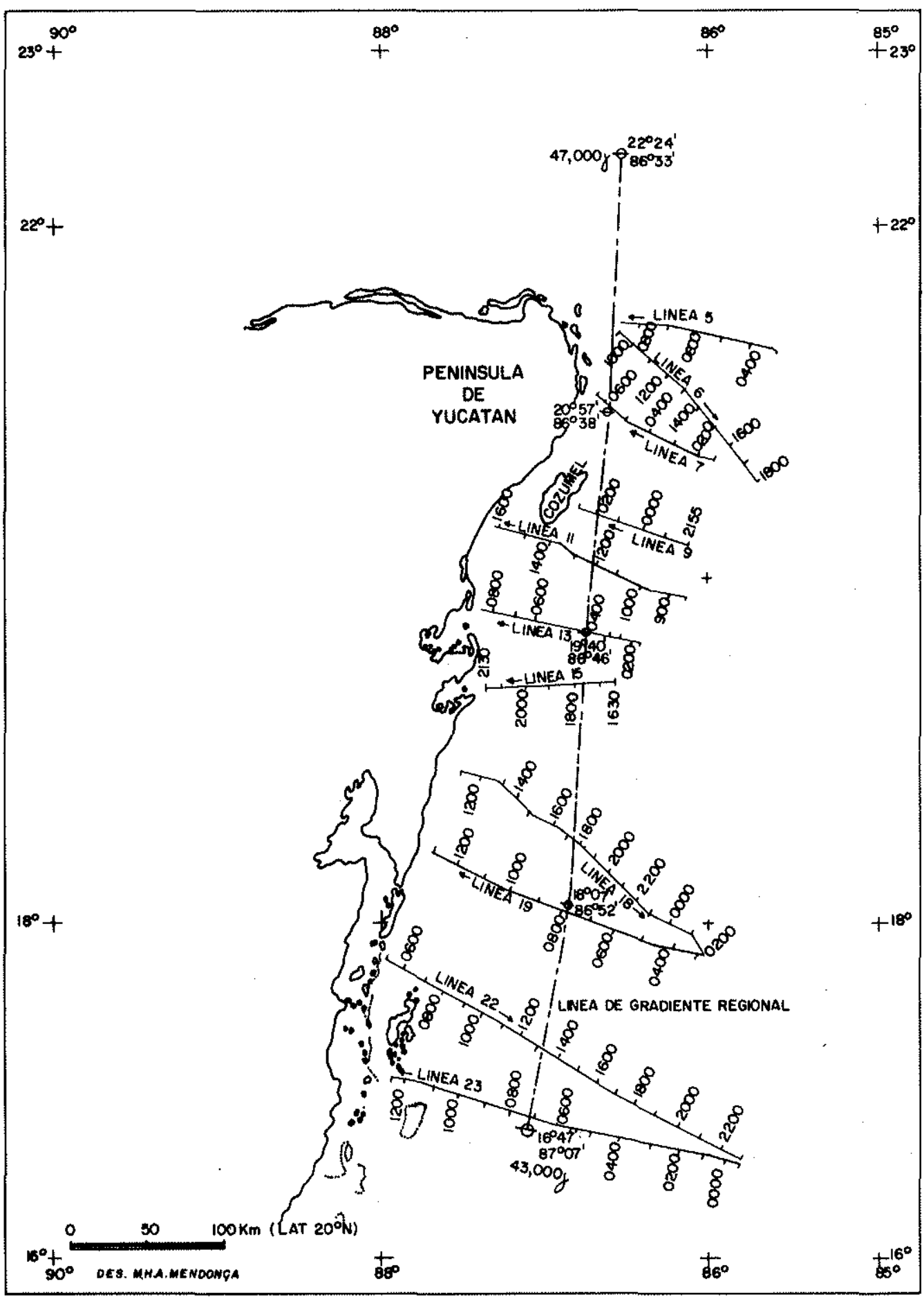

Figura 1 - Transectos del leg 2 utilizados en la interpretación de la porción occidental de la zona del Caribe 
Los perfiles del LEG 2 comprenden más de $4.450 \mathrm{~km}$ de extensión con un total de 32 transectos. En la presente investigación fueron seleccionados y analizados once transectos que suman más de $1.500 \mathrm{~km}$ de información geofísica contínua a manera de perfiles. Las observaciones geofisicas incluyen medidas de gravimetría, magnetismo, reflexión acústica contínua así como de la batimetría.

Tomando como base algunos estudios anteriores, en esta ocasión, se pretende prow porcionar un mayor entendimiento de la tectónica con la aportación de parámetros geofísicos más precisos y su adecuada interpretación y correlación con las hipótesis prevaw lecientes.

INVESTIGACIONES ANTERIORES Desde de la primera síntesis del Caribe (Suess, 1885) esa región y áreas adyacentes ha sido estudiada no sólo desde el punto de vista netamente tectónico sino también como área de interés económico. Particularmente, las áreas de la Cresta de Caymán y Fosa de Bartlett (o Caymán), han sido motivo de diversos estudios, pues se trata de estructuras críticas en cualquier hipótesis sobre el origen del Caribe.

Ewing et al. (1960) en base a perfiles de refracción profunda presentó un modelo de la corteza a través de todo el Caribe; Bowin (1968) realizó estudios de batimetría, gravimetría y magnetismo de la Cresta de Caymán y Fosa de Bartlett; Dengo (1969) investigó sobre las relaciones tectónicas entre América Central y el Caribe; Arden Jr. (1969) emitió una idea sobre la história geológica del Banco de Nicarágua; Molnar y Sykes (1969) limitó la Placa del Caribe en base a estudios de mecanismos focales; Baie (1970) sugirió una posible unión estructural entre Cuba y la Península de Yucatán; Fahlquist y Davies (1970) estudió la Cresta de Caymán a través de perfiles de reflexión contínua y magnetismo; finalmente, P. Malin (comunicación escrita) presentó un modelo para la Cresta de Caymán.

REGOLEGGION Y REDUCGION DE DATOS El barco utilizado en el crucero LEG 2 fue el buque oceanográfico UNITEGEO-1 (Vedder et al., 1971). Para la navegación se hizo uso de un sistema basado en observaciones por satélite, suplementado por LORAN y RADAR. La precisión en la determinación de una fija por satélite se estima en $180 \mathrm{~m} \mathrm{y}$ en el sistema de navegación por RADAR el error promedio de posición, en la identificación de puntos de control, es de 0.5 millas náuticas $(925 \mathrm{~m})$. La velocidad de crucero oscilaba alrededor de 8.5 nudos durante casi todo el levantamiento marino.

Se empleó un gravímetro marino marca LaCoste \& Romberg con plataforma estabilizadora y un sistema de graficador contínuo. En condiciones favorables, la precisión en las medidas gravimétricas es de $\pm 2 \mathrm{mgal}$, excluyendo los errores en la navegación. En la presente investigación se hizo uso de la fórmula de Stacey (1969) para calcular la gravedad teórica. Cabe mencionar que las medidas gravimétricas también se corrigieron por el efecto de Eötvös.

Para obtener la intensidad magnética total se hizo uso de un magnetómetro de precesión nuclear marca Varian. Se cree que se logró alcanzar una precisión de $\pm 1 \gamma$.

Los perfiles acústicos de reflexión fueron obtenidos con un sistema de Sparker, cuya máxima energia producida llegaba a alcanzar hasta $220 \mathrm{KJ}$ en conjunto. La energia podría ser cambiada según el interés resolución o penetración. En batimetria se empleó un equipo de penetración profunda para el perfilaje de la topografia del fondo marino. La profundidad del agua se obtuvo en segundos (two-way travel time) y se corrigió en base a las tablas de Matthews. Los datos de profundidad fueron computados contínuamente.

Los programas para la computadora empleados en este estudio son los que generalmente utiliza el Departamento de Exploración del Instituto de Geofísica de la UNAM en sus diferentes 
investigaciones. La computadora empleada fue una Burroughs B-6,500 del Centro de Investigaciones de Matemáticas Aplicadas y Sistemas (CIMASS) de la UNAM. En total se utilizaron cinco programas diferentes para reducción e interpretacion, tomando como base los algoritmos de Talwani et al. (1959), Corbató (1965) y algunos otros realizados por los propios investigadores del citado Departamento.

ASPECTOS GEOLOGICO-GEOFISICOS Batimetria Los rasgos físiográficos más importantes en el área son: el Canal de Yucatán, ubicado en el norte; al centro aparece la Cuenca de Yucatán y más hacia el sur las estructuras que forman la Cresta de Caymán y la Fosa de Bartlett (Figura 2).

El Canal de Yucatán és una depresión abrupta, cuya profundidad alcanza cerca de los $2.000 \mathrm{~m}$, presentando una pendiente un poco más suave en dirección al continente mexicano. En la parte oriental del área, es decir, el borde occidental de México y América Central, la zona comprendida entre las cotas batimétricas de $1.000 \mathrm{~m}$ es relativamente paralela al margen del continente. En direción hacia el occidente, la topografia continúa suavemente descendente hasta encontrar um cierto número de altos batimétricos que originam dos crestas, descritas por Baie (1970) como Cresta Interna y Cresta Externa. Estas dos escarpas submarinas están separadas por una depresión que origina várias pe.

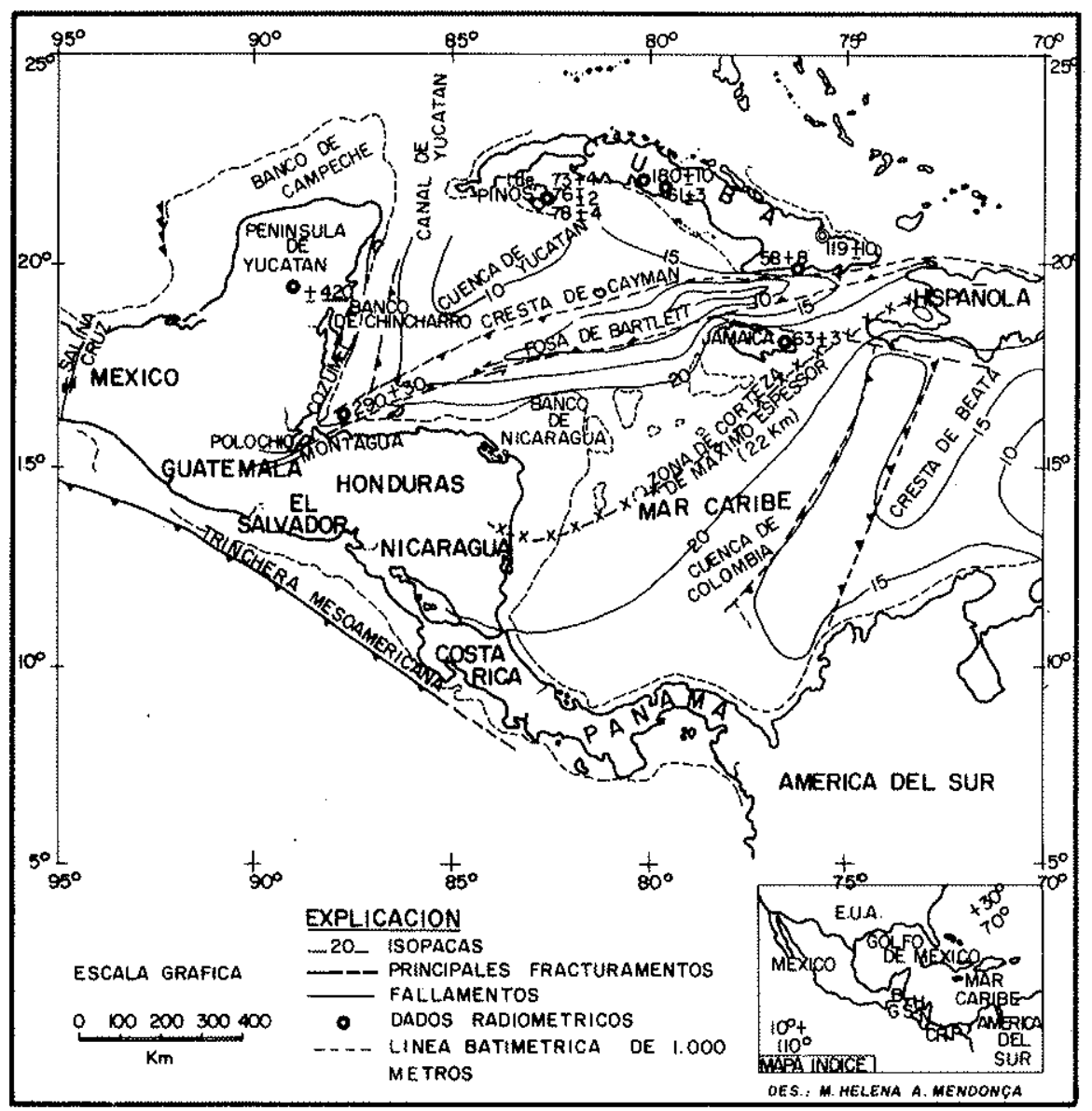

Figura 2 - Tectónica regional de la zona del Caribe y areas adyacentes (basado en Arden Jr., 1969) 
queñas cuencas. La Cresta Interna tiene una dirección general paralela al borde continental. Sin embargo, la Cresta Externa en las proximidades de la Isla de Cozumel, cambia su alineamiento hacia el NE y se extiende en dirección de la Isla de Cuba. La parte oriental de esta cresta se profundiza abruptamente originando una escarpa de fuerte pendiente que corresponde al límite occidental de la Cuenca de Yucatán. Esa gran depresión es la más prominente característica en toda el área y posee una forma alargada de dirección paralela a la Cresta Externa. La máxima profundidad de la Cuenca de Yucatán se localiza próxima a la parte central $(-4.500 \mathrm{~m})$.

La Cresta de Caymán es una estructura lineal contínua con dirección general NE-SW, cuya cima posee una forma achatada y está delimitada por dos escarpes de fuertes pendientes. Datos recientes sugieren que dicha estructura continúa en dirección hacia Honduras Británicas, pero grandemente suavizada ( $\mathrm{P}$. Malin, com. escrita). El lado occidental de la cresta se eleva alrededor de $1.500 \mathrm{~m}$ hasta $2.000 \mathrm{~m}$ sobre el nivel del fondo marino. La parte opuesta a esta dirección posee una pendiente bastante suave y larga, llegando a profundidades abisales $(-6.000 \mathrm{~m})$ precisamente donde se inicia la Fosa de Bartlett. La parte meridional de esta estructura limita el Banco de Nicarágua que ya se encuentra fuera del área investigada.

Marco tectonofisico La descripción de la geología del área estudiada y en particular la parte continental de América Central, se basa, principalmente, en los trabajos de Dengo (1968, 1969). El límite continental del área comprende parte de América Central que se ha definido como un área terrestre y de plataforma continental, la cual se extiende desde el Istmo de Tehuantepec en México y continúa en dirección este y SE hasta las tierras bajas de Atrato en Colombia.

América Central está dividida en dos unidades geológicas principales, cuyo límite se sitúa en la parte Central de Nicarágua. En la unidad norte, el basamento se caracteriza por la presencia de rocas cristalinas pertenecientes al Paleozóico, que a su vez son cubiertas por rocas sedimentarias del Paleozóico Superior (Pensilvaniano y Pérmico) en una franja angosta. Las rocas Paleozóicas se localizan subyaciendo a grandes extensiones de rocas sedimentarias mesozóicas. Sobre el basamento de la Península de Yucatán aparecen sedimentos con más de $3.000 \mathrm{~m}$ de espesor. Estas rocas fueron depositadas en su mayor parte desde el Cretácico Superior hasta el Eoceno Medio, en forma casi contínua e ininterrumpida (Dengo, 1968). Una muestra del basamento obtenida en el Pozo Yucatán N. ${ }^{\circ} 1(-3.178 \mathrm{~m})$, indica una edad probable de 420 m.a. (Bass y Zartman, 1969).

En la parte sur de América Central, las rocas más antíguas pertenecen al Mesozbico y están representadas por volcánicas básicas e hipabisales, que subyacen a rocas sedimentarias marinas y volcánicas del Cretácico Superior, Terciário y Cuaternário (Dengo, 1968).

El mapa de isopácas dado a conocer por Arden Jr. (1969) en relación al acomodo interior de las áreas adyacentes al Mar Caribe, proporciona un magnífico esquema sobre el comportamiento de la corteza para esa región (Figura 2). Bajo el Oceano Atlántico y ya en las proximidades del Caribe, la corteza tiene un espesor de 4 a $5 \mathrm{~km}$. En la Fosa de Bartlett el espesor mínimo medido fue de sólo $4.1 \mathrm{~km}$. En el centro de la Cuenca de Venezuela es de $5 \mathrm{~km}$. La corteza más espesa (22 km) ocurre en el cinturón de las Islas Antillanas y en la parte sur del Banco de Nicarágua. Worzel y Shurbet (1955) indica en su sección sísmica, levantada en el Banco de Campeche (Golfo de México), un espesor de la corteza de 18 a $25 \mathrm{~km}$ en el área de la plataforma continental y que este espesor aumenta hacia el continente en la Península de Yucatán.

Hess (1960) afirmó que al norte de la 'Trinchera de Puerto Rico y en el mar abierto, fuera del Caribe, la velocidad sísmica debajo de la Discontinuidad $M$ es de cerca de $8 \mathrm{~km} / \mathrm{seg}$. 
Al sur de la Trinchera y bajo el Caribe la velocidad es de $7.4 \mathrm{~km} / \mathrm{seg}$. Esta disminución de velocidad se debe a serpentinización parcial de un manto de peridotita. Pruebas de laboratório efectuadas por el propio Hess confirman esta hipótesis. En la Isla de Puerto Rico, las peridotitas serpentinizadas ocurren como núcleos de los grandes anticlinales y son discordantes abajo de rocas sedimentarias y volcánicas del Cretácico Tardío. En contraste, los anticlinales mayores de Venezuela poseen un núcleo granítico. Este facto parece indicar que las Antillas fueron formadas a partir de una corteza oceánica, mientras que las últimas de la costa provienen de una corteza siálica. Los datos, tanto de espesor de corteza, densidad y velocidad sísmica, sugieren que las Antillas descansan sobre una corteza de composición intermédia, entre la siálica de los continentes y la delgada y densa de los oceanos.

Dentro de las cuencas de la zona Golfo de México-Caribe no se ha podido demonstrar la existencia de rocas más antíguas que el Jurásico (Arden Jr., 1969). Donnelly (1964) indica que las rocas más antíguas de Puerto Rico e Islas Vírgenes fueron depositadas aparentemente sobre una corteza oceánica y que no existe evidencias de una corteza siálica donde se podrían depositar unidades más antíguas. Según Spencer (1969) la geología general de las Grandes Antillas (Cuba, Hispañola, Puerto Rico y Jamaica) posee gran similitud, aunque existen discrepancias en ciertos detalles, además de que las rocas más antíguas en todos los casos coinciden en una edad Mesozóica. Para las rocas más antíguas de Cuba, representadas por un batolito (Sierra de Trindad), Meyerhoff et al. (1969) propuso una edad de 180 m.a. (Jurásico Inferior). La Figura 2 indica la localización y edades de varias rocas en diferentes puntos del Caribe y en la parte continental de América Central.

Diversas pruebas geofísicas parecen indicar que parte de la región del Caribe está constituida por una placa distinta, rígida, con desplazamiento propio, ubicada adentro de la gran Placa Americana. Mapas de epicentros (Molnar y Sykes, 1969) en la región del Caribe y zonas adyacentes muestran una concentración de actividad sísmica bordeando el Caribe y una ausencia de sismos adentro de la propia cuenca del Caribe. Este cinturón de sismicidad que circunda el área posiblemente defina las márgenes de la Placa o Sub-Placa del Caribe. Molnar y Sykes (1969) basandose en estudios de los mecanismos focales, sugiere que el movimiento de la Placa del Caribe se efectúa en dirección este, con respecto a la Placa Americana. El desplazamiento a lo largo de estas dos placas se realiza a través de los planos profundos de la Fosa de Bartlett en dirección E-W. El principal movimiento diferencial se produce paralelamente a esta zona y perpendicular al Arco de las Pequeñas Antillas.

CORRELACION DE LA INFORMACION Reflexión acústica Gran parte de los perfiles sísmicos (Figuras 3 y 4) que cubren el área de interés indican que se tiene un basamento bastante irregular, cubierto en muchas áreas por estratos sedimentarios casi sempre ho* rizontales. El término basamento es empleado en forma generalizada para indicar un reflector constituido de rocas con un mayor grado de densidad donde se verifica un incremento de la velocidad de las ondas compresionales.

En los perfiles referentes a la Cuenca de Yucatán se puede distinguir, en algunas áreas, tres unidades distintas de reflexión. La primera, la más profunda, está representada por el basamento, identificado por altos y bajos, fácil de se distinguir. Inmediatamente arriba del basamento se tiene una unidad caracterizada por un horizonte de reflexión poco coherente, de espesor variable para tiempos de 0.0 y $0.6 \mathrm{seg}$. En el topo de la secuencia, la tercera unidad está representada por una capa de reflexión bastante potente, con estratificación horizontal. Ese horizonte, generalmente de espesor constante, en la. Línea 18 (Figura 3) llega a presentarse con una extensión de $90 \mathrm{~km}$. La división de los reflectores 


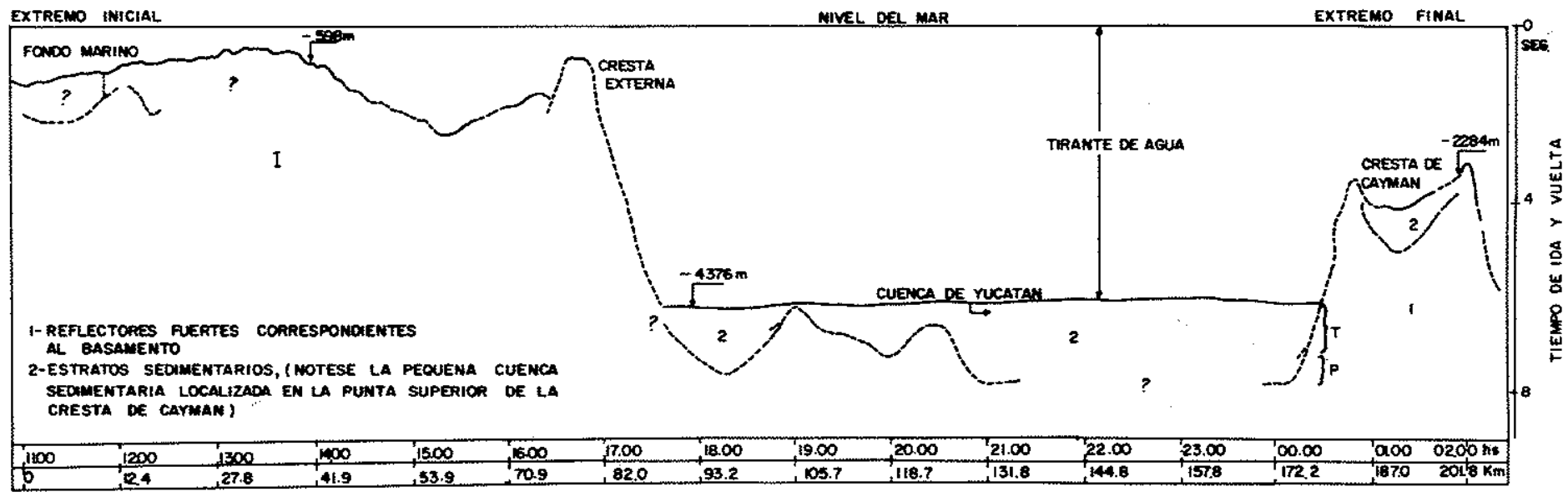

Figura 3 - Interpretación del perfil de reflexión acústica - linea 18

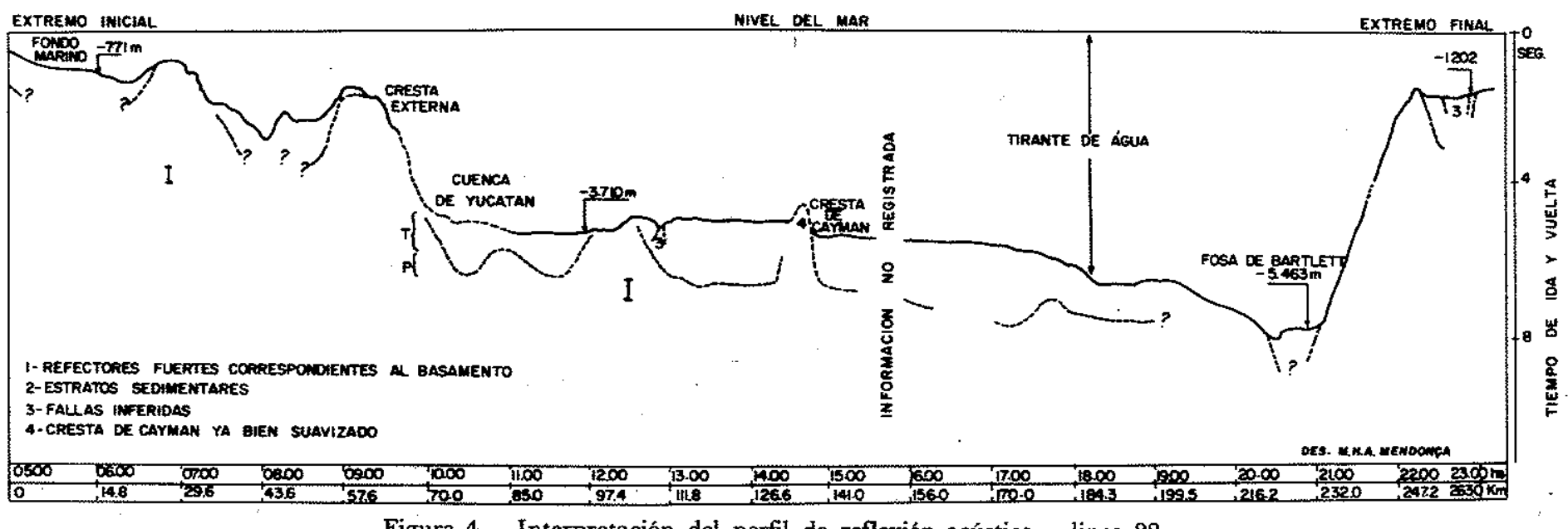

Figura 4 - Interpretación del perfil de reflexión acústica - linea 22 
sedimentarios en dos unidades distintas está basado en conclusiones de Falhquist y Davies (1971) y P. Malin (com. escrita). Estos investigadores clasifican la primera unidad como sedimentos de origen pelágico $(\mathrm{P})$ y la segunda como una secuencia de productos de corrientes de turbidez (T).

En la Línea 18 (Figura 3), la Cuenca de Yucatán se encuentra entre dos estructuras positivas representadas por la Cresta Externa y la Cresta de Caymán. Esa última, en su parte superior ( $\mathrm{km} 185$ y 200) presenta una topografia muy peculiar, esto es, dos picos que parecen circundar una pequeña cuenca sedimentaria en la cima de la cresta. La porción superior de los picos podría representar arrecifes de coral, conforme a lo sugerido por Banks y Richards (1969).

De la Cresta Externa, límite occidental de la Cuenca de Yucatán en dirección al continente, la división de los horizontes sedimentarios en las dos unidades, anteriormente descritas, se dificulta. La pequeña amplitud de las capas sedimentarias en estas áreas, quizá podría explicarse por la presencia de la Cresta Interna. Dicha estructura funcionaría como una barrera de contención para los sedimentos terrígenos ahí presentes.

El examen minucioso de los registros sísmicos desde un punto de vista tectónico indica, principalmente, en las Líneas $6,11,13$ y 18 que la fuerte pendiente que conecta el flanco oriental de la Cresta Externa con el fondo de la Cuenca de Yucatán parece estar asociado a efectos de afallamiento. En los registros muchas fallas son perfectamente delineadas pero sus extensiones a profundidad son dificiles de discenir, principalmente, por el efecto de reflexiones múltiples. La mayoria de estas fallas aparentemente se restringen a las capas sedimentarias. Las fallas que están conectando sedimento-embasamento son menos frecuentes y al mismo tiempo dificiles de determinar.

Potenciales En el perfil de la Línea 6 (Figura 5) el máximo valor de la anomalía de aire libre se encuentra en su extremo oriental y el valor mínimo en el $\mathrm{km} 55$. Ese último punto corresponde al centro de una gran anomalía que se incrementa en ambas direcciones laterales. El bajo correspondiente a esa anomalia se ha interpretado como el hundimiento de un gran bloque limitado en sus extremos por las Crestas Interna y Externa. El fuerte gradiente positivo en dirección hacia el este, podría explicarse por la presencia de la Cuenca de Yucatán, a partir del km 120, donde habría una corteza de característica más oceánica. En el km 26, el perfil presenta un bajo simétrico com $14 \mathrm{~km}$ de ancho correlacionáble con el flanco occidental de la Cresta Interna.

El perfil magnético de esta línea presenta un dipolo notable en su extremo occidental con longitud total de $50 \mathrm{~km}$. Se intuye que la dipolaridad refleja efectos anómalos de la Cresta Interna correspondiente a una falla o material ajeno. Probablemente ese material fluyó a través de la debilidad ocasionada por el hundimiento de un bloque tal y como se refirió en el párrafo anterior. A lo largo del perfil aparecen aún dos pequeños altos magnéticos de poca amplitud localizados en los $\mathrm{km} 70$ y 85 . Estas anomalías probablemente son producidas por rugosidad del basamento a profundidad.

Cuanto la anomalía de aire libre de la Línea 15 (Figura 6) el facto interesante es su carácter ascendente a partir del km 20 hacia el oriente. La presencia de este gradiente se atribuye a un rápido adelgazamiento de la corteza siálica y por consiguinte de un ascenso de material basáltico. El punto de cambio entre eses dos gradientes corresponde al inicio de una acentuada pendiente en la topografia del fondo oceánico y que conecta el princípio de la Cuenca de Yucatán. En esta zona los sedimentos de la cuenca se cree que descansan sobre una corteza constituida de material basáltico.

El perfil magnético de la Línea 15 presenta, en su porción occidental, un bajo con forma de cuña cuyo valor medido alcanza una centena de gamas. A partir del $\mathrm{km} 10$ existe 


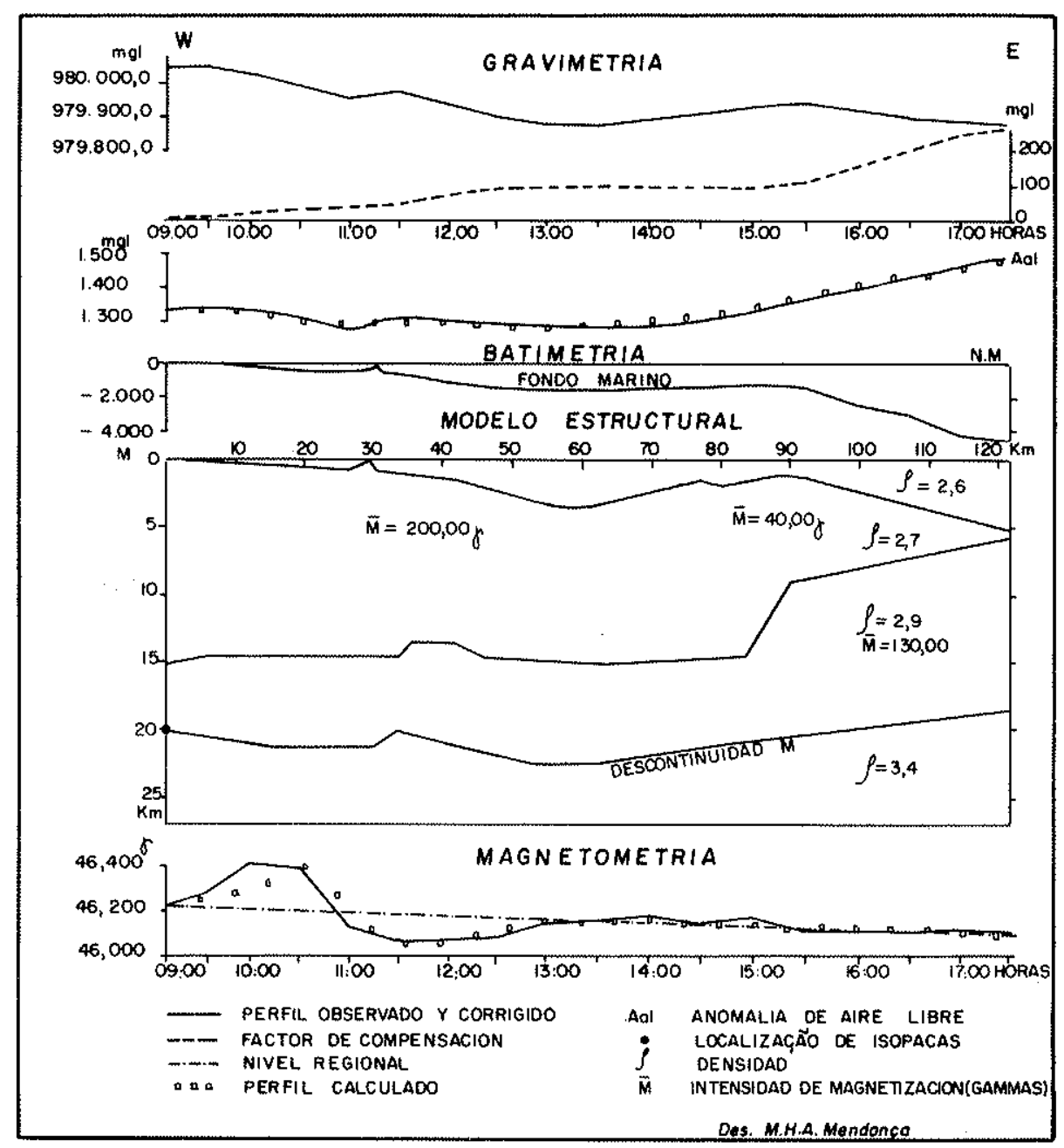

Figura 5 - Interpretación geofisica (linea 6)

un gradiente positivo y al que parece esa anomalía guarda estrecha relación con la topografia del fondo marino. Del km 35 al 68 el gradiente, en dirección este, se torna más suave. En dicha sección las amplitudes anómalas alcanzan valores del orden de $20 \%$. A partir del $\mathrm{km}$ 68, en dirección hacia el oriente, existe un cambio brusco del gradiente; dicho decremento coincide con el surgimiento de la corteza basáltica de acuerdo al modelo estructural propuesto. Probablemente las propriedades de magnetización del material basáltico causen el cambio en el gradiente magnético.

El perfil gravimétrico de la Línea 22 (Figura 7) presenta dos aspectos muy interesantes y distintos a los de las Líneas 6 y 15 . El más sobresaliente es un escalón resultante de un fuerte gradiente en el $\mathrm{km} \mathrm{50}$; el segundo aspecto se refiere a un máximo de forma simétrica de aprosimadamente $110 \mathrm{~km}$ de ancho. El salto o escalón probablemente esté reflejando un repentino adelgazamiento de la corteza siálica, directamente abajo de la Cresta Externa. En dirección este, los valores de la anomalía de aire libre continúan aumentando, pero en forma más lenta; esto se debe a la presencia de una corteza granítica cada vez más delgada. Este perfil intercepta la Cuenca de Yucatán en su extremo final y se cree que la estruc- 


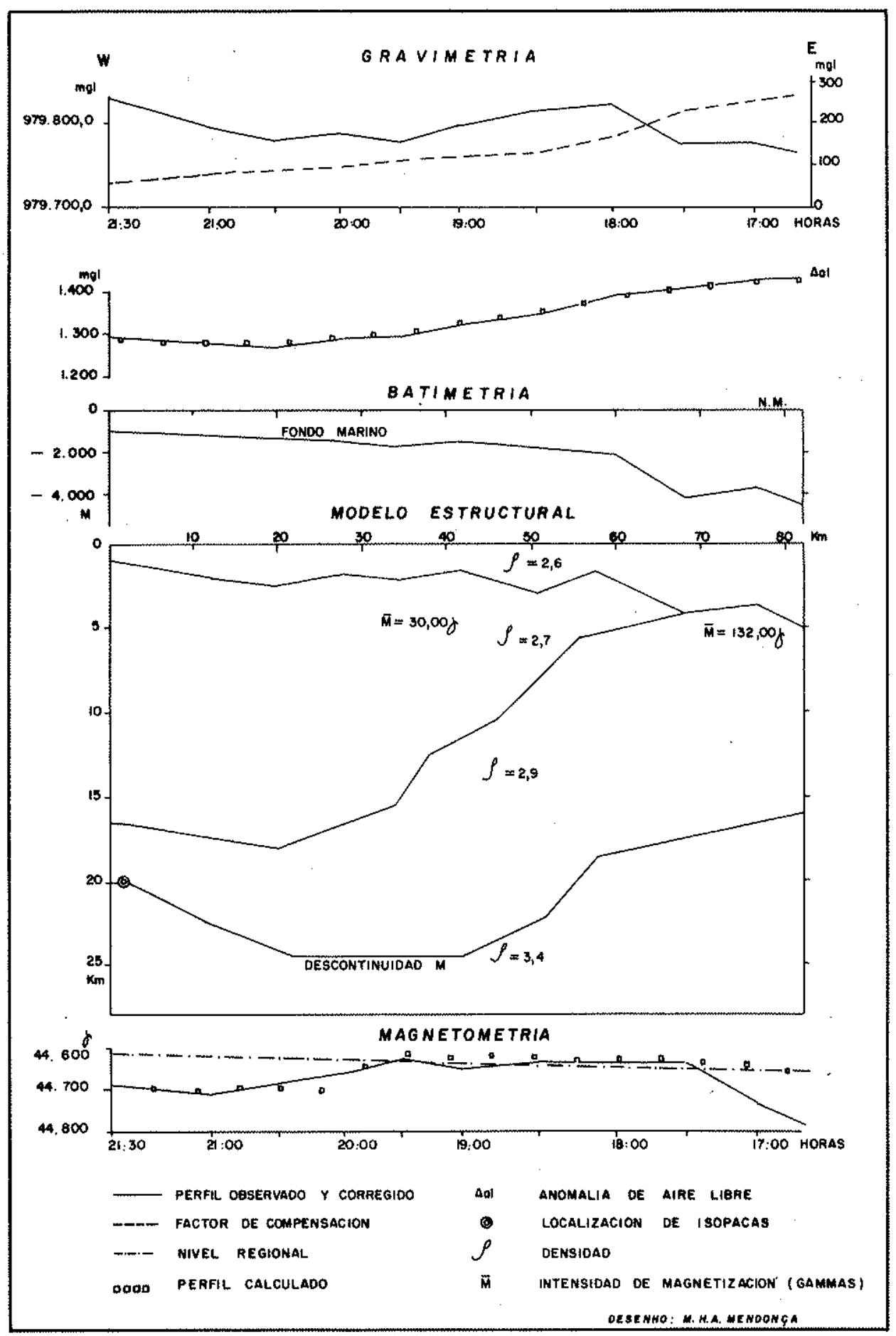

Figura 6 - Interpretación geofisica (linea 15) 


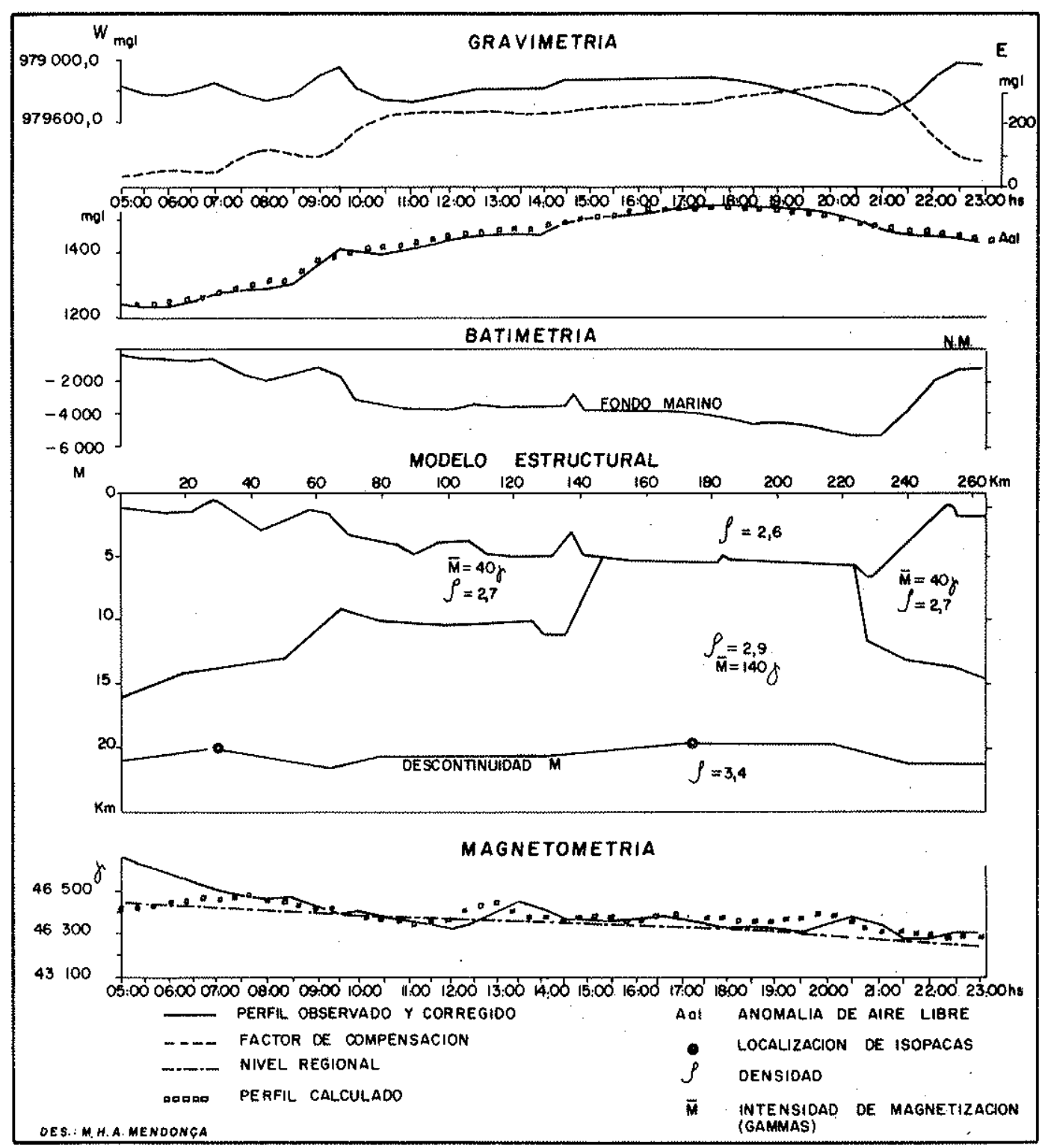

Figura 7 - Interpretación geofisica (linea 22)

tura está situada sobre una corteza granítica, pero bastante delgada. La gran anomalía ubicada entre los km 130 y 240 se correlaciona con la Fosa de Bartlett y áreas adyacentes. El carácter de esta anomalía sugiere una posible interpretación para el área de la fosa como localizada sobre una corteza constituida por material basáltico. Después del $\mathrm{km} 240$ el gradiente se torna levemente decreciente y este comportamiento concuerda con la idea de que la Línea 22 se encamina nuevamente hacia el continente.

En cambio la anomalía magnética de la Línea 22 presenta en su extremo occidental un pronunciado gradiente en dirección hacia el continente; en los $\mathrm{km} 120$ y 225 se tiene dos altos magnéticos con extensiones de 20 y $25 \mathrm{~km}$. Aparentemente existe una estrecha 
relación con la elevación del terreno debido a la Cresta de Caymán y al declive occidental de la Fosa de Bartlett. Sin embargo, es interesante observar que las anomalías aparecen desplazadas hacia el occidente con relación a los rasgos topográficos. Se intentaron otros modelos con distintos vectores de magnetización y diferentes intensidades, pero la falta de superposición persistió. Si se asume que la topografia del basamento es correcta en base a los registros de reflexión contínua y que se corrobora con la compensación de la gravimetría, la correlación podría fundamentarse en parámetros de magnetización remanente en las rocas del área. En el modelo bidimensional no se toma en consideración las variaciones normales de las rocas en el sentido transversal al cuerpo. Este hecho no puede controlarse con gran efectividad y la influencia es mucho mayor en las variaciones del campo magnético que en las del campo gravífico.

DISCUSION MODELO ESTRUGTURAL Los modelos estructurales bidimensionales (Figuras 5, 6 y 7) se construyeron tomando en cuenta la batimetría, los registros sísmicos y las variaciones de los campos gravífico y magnético. Estos modelos son constituidos por tres cuerpos con propriedades fisicas distintas. El primer cuerpo estaria simulando una capa granítica, el segundo una capa basáltica y el tercero un material del manto superior. El cálculo del campo teórico, gravimétrico y magnético, producido por el modelo, se evaluó a intervalos regulares de $5 \mathrm{~km}$. Los resultados obtenidos se compararon con los valores observados y corregidos hasta que se obtuvo una superposición aceptable para la precisión obtenida.

En gravimetría los valores empleados para los diferentes horizontes, o cuerpos, fueron de $2.7 \mathrm{gr} / \mathrm{cc}$ para el cuerpo granítico, $2.9 \mathrm{gr} / \mathrm{cc}$ para el basalto y de $3.4 \mathrm{gr} / \mathrm{cc}$ para el material más denso del manto superior.

La intensidad de magnetización para el cuerpo granítico fue de $20-40 \gamma$ y para el cuerpo basáltico varió de 130 a $140 \%$.

RESUltados Durante el rompimiento de los continentes Americano y Afro-Europeo, se cree que la separación estuvo acompañada de una ligera rotación. La hipótesis presentada por Freeland y Dietz (1971) sugiere que la separación de las Américas del Norte y Sur se efectuó en forma simultánea con la apertura del Golfo de México, ocurrida posiblemente en el Triásico Tardio. La teoria concuerda con un núcleo para las Grandes Antillas formadas a partir de una espesa secuencia sedimentaria. Esta cadena de islas daría lugar a neo-cratones originados de una corteza intermedia. Además, el Mar Caribe y el Golfo de México eran pequeñas cuencas localizadas entre áreas intracratónicas.

Freeland y Dietz (1971) indica que el área de la Península de Yucatán, constituída por un antíguo cratón, en la actualidad no ocupa su posición original, ya que ésța se localizaría más al NW. Algunos datos geológicos y geofísicos sugieren la existencia de una corteza distinta para esta área continental. Las evidencias geológicas pueden complementarse con evidencias tectónicas, las cuales se inclinan por una individualización, tanto de corteza continental, como de corteza oceánica.

A grandes rasgos, el bloque de Yucatán puede limitarse en base a las zonas de debilidad que lo circundan actualmente. En esta forma el límite hacia la porción sur estaría dado por la extensión con rumbo hacia el continente del sistema de falla de la Fosa de Bartlett. Esta zona de falla, en dirección occidental, se correlacionaría con las fallas de Polochic y Montagua (Figura 2), las cuales tienen un rumbo, aproximadamente, E-W en su parte central. Si se correlacionan algunas lavas de ambos lados de la falla, el desplazamiento sería de $20 \mathrm{~km}$ (McBirney, 1963). En el oriente de la Península de Yucatán, la debilidad en la corteza podría representarse por el flanco occidental de la Cresta Externa. La inter- 
pretación de los registros sísmicos y anomalias gravimétricas y magnéticas, en base a los modelos que justifican los campos, sugieren la zona de la Cresta Externa como un área de fallamiento profundo. Probablemente esta zona podría corresponder al límite de separación entre la corteza continental y la intermédia bajo el Caribe. En el lado occidental del cráton de Yucatán se encuentra la falla de Salina Cruz con dirección NE-SW; ese fracturamiento con más de $350 \mathrm{~km}$ de extensión corta el Istmo de Tehuantepec. Investigaciones sísmicas sugieren su prolongación en dirección norte, en posición directa hacia el Golfo de México (Viniegra, 1971). En la parte superior occidental de la Península de Yucatán el contacto del Banco de Campeche con la porción SW del Golfo de México, se hace a lo largo de un fuerte escarpe también con dirección general N-S. Esta pendiente abrupta tendría una extensión mayor de $100 \mathrm{~km}$ si se considera un desnivel de $2.000 \mathrm{~m}$ entre las curvas batimétricas de 500 y $2.500 \mathrm{~m}$. Al correlacionar la información tectónica para un desnivel de $1.000 \mathrm{~m}$, la extensión del escarpe debería ser mayor de $200 \mathrm{~km}$. Investigaciones recientes del Departamento de Exploración del Instituto de Geofísica de la UNAM (Moore y Del Castillo, 1972; J. H. Sandoval, com. verbal) revelan que las isopacas en esta área se relacionan con una corteza con tendencia a un rápido adelgazamiento en dirección al interior del Golfo de México. Aparentemente el comportamiento estructural de la corteza

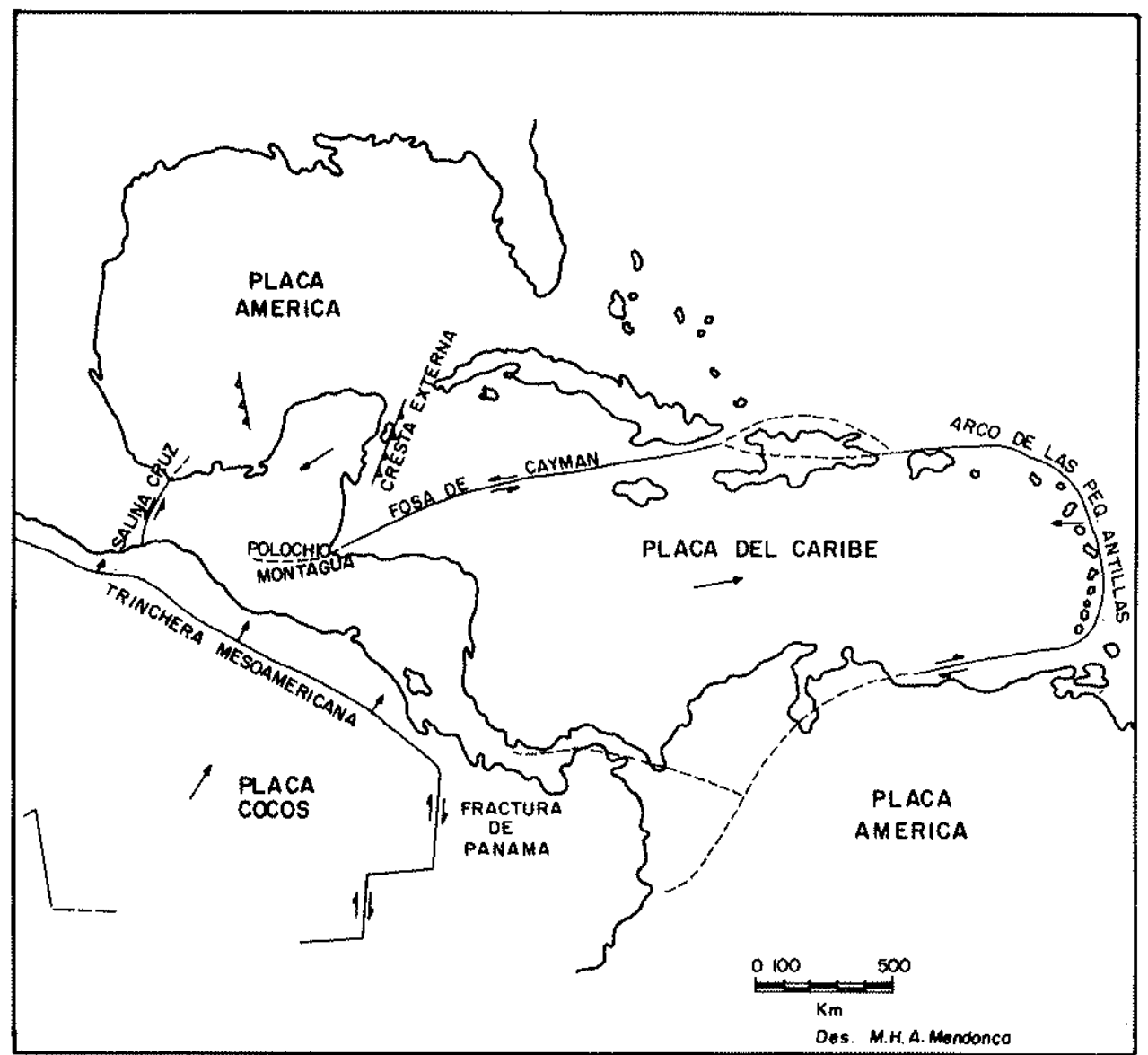

Figura 8 - Placas litosféricas y sus movimientos en la zona del Caribe y areas adyacentes (basado en Molnar y Sikes, 1969) 
en esta área indica un contacto de fuertes contrastes, implicando la existencia de una amplia sutura, entre la unidad representada por el cratón de Yucatán y la corteza cuasi-océanica debajo del Golfo de México. Además de los factores citados, otro fuerte argumento de apoyo a la suposición de la existencia del cratón de Yucatán es el comportamiento geológico de la región. Se ha referido que América Central está dividida en dos grandes unidades que son producto de dos histórias geológicas distintas.

Los registros sísmicos en el área de la Fosa de Bartlett muestran que los sedimentos no sufrieron una gran perturbación. De esta observación se puede concluir que el principal fallamiento ocurrió con antelación a la mayor parte de los depósitos sedimentarios en el área. Si se acepta la hipótesis de que la estructura actual de la Fosa de Bartlett es realmente consecuencia de la deriva, los sedimentos más antíguos en esta área probablemente sean Mesozóicos. El modelo estructural presentado para la Línea 22 (Figura 7) se construyó en base a la idea de que la Fosa de Bartlett es producto de esfuerzos tensionales en la corteza (Bowin, 1968).

En apoyo a la discusión anterior, la Figura 8 muestra en forma esquemática los movimientos diferenciales de diversos bloques corticales a través de los diferentes fracturamientos en el área.

CONCLUSIONES Al tratar de establecer una correlación de la información tectonofisica existente con el análisis de modelos y datos geofísicos aportados por este estudio, se pueden hacer algunos comentários de interés para la zona del Caribe.

Algunos de los modelos propuestos y especialmente el de la Línea 15, representan una típica interación de una corteza continental con una océ́nica. Si se admite la existencia de una corteza de característica intermedia debajo del Caribe, se podría sugerir que el flanco oriental de la Cresta Externa representa a su vez el límite, aproximado, de las cortezas continental y intermedia.

La sección comprendida entre los km 30 y 90 en la Línea 6 (Figura 5) sugiere la existencia de un gran bloque hundido como consecuencia de esfuerzos tensionales. Este hundimiento es de gran magnitud, tanto en extensión como en profundidad y además, existen en el área otras evidencias de estructuras de esta naturaleza. Posiblemente estos esfuerzos tensionales provengan de movimientos relativos entre placas litosféricas existentes en la zona o en particular, del movimiento inicial y posterior acomodación del cratón de Yucatán.

El modelo sugerido para la Línea 22 (Figura 7) sigue la idea que la Fosa de Bartlett es una estructura resultante de esfuerzos tensionales aunada a una subida simultánea de material denso, proveniente de mayores profundidades.

El análisis de los registros sísmicos indica la presencia de un basamento bastante irregular cubierto por sedimentos con estratificación horizontal. Esta situación parece implicar que durante y después de la época de depositación de la mayoría de los sedimentos el área no sufrió grandes perturbaciones. Los sedimentos existentes en las inmediaciones de la Fosa de Bartlett también presentan esta característica. Si se admite que el principal o último movimiento que dió origen a dicha fosa ocurrió en el Mesozóico, probablemente los sedimentos más antíguos en esta área sean también de esa edad. Determinación radiométrica y una correlación paleontológica de la edad de estos sedimentos sería muy valiosa en la explicación de la historia geológica del área.

Las evidencias citadas anteriormente complementadas con estudios realizados por otros investigadores, permiten hacer una conclusión general del área que sería la siguiente:

a. Evidencias geológicas y geofisicas en la parte continental de México y porción de América Central comparados con los de la región del Caribe, permiten distinguir y 
separar estas dos grandes zonas por cuanto a los aspectos de edad y origen. Entonces el contacto, aproximado, entre las dos cortezas de características distintas se propone a lo largo del área de la Gresta Externa. Esto es una opinión contrária a la hipótesis presentada por Baie (1970) de que probablemente existiría una unión estructural entre Cuba y Amém rica Central.

b. Los mapas de epicentros y estudios de mecanismos focales demuenstran la existencia de la Sub-Placa del Caribe con movimiento propio, en dirección este, ubicada dentro de la Placa Americana. La sub-placa está protegida de la destrucción por zonas de subtracción; al este representada por el Arco de las Antillas y al oeste por la Trinchera Mesoamericana (Molnar y Sykes, 1969).

c. Según las evidencias geológicas y tectónicas es probable que el bloque de Yucatán sea realmente un antíguo cratón que actualmente no ocupa su posición original, ya que ésta se encontraría más al occidente.

d. La región del Caribe y áreas adyacentes se cree que tienen su origen en el Meso" zóico como resultado de la fisura y subsecuente deriva de los continentes Americano y Afro-Europeo.

AGRADECIMIENTOS Los arreglos iniciales para la participación de los investigadores de la UNAM en el proyecto IDOE fueron encauzados por los Drs. Agustín Ayala G., Coordinador de Investigación en la UNAM; y Louis E. Garrison, Jefe de Geologia Marina del USGS en Corpus Christi, Tex. Se agradece la ayuda prestada en compilación y procesado a los miembros del Departamento de Exploración del Instituto de Geofisica de la UNAM, especialmente las sugerencias de A. Cominguez y L. Mendive, el empeño y dedicación de J. Sandoval, M. A. Calderón y M. Rivas T.; asi como las discusiones e intercambio de información con colegas nacionales y del USGS, en. especial con el Dr. John G. Vedder y su grupo de asistentes en Menlo Park, California.

\section{BIBLIOGRAFIA}

ARDEN, JR., D. - 1969 - Geologic history of the Nicaraguan Rise. Gulf Coast Assoc. Geol. Soc. vol. V, n. ${ }^{\circ}$ XIX, pp. 295-309

BAIE, L. F. - 1970 - Possible structural link between Yucatán and Cuba. Am. Assoc. Petroleum Geol. Bull. vol. 54, n. ${ }^{\circ} 11$, pp. 2 204-2 207

BANKS, N. G. e RICHARDS, M. L. - 1969 - Structure and bathymetry of western end of Bartlett Trough, Garibbean Sea. Am. Assoc. Petroleum Geol. Mem. 11, pp. 221-228

BASS, M. N. e ZARTMAN, R. E. - 1969 - The basement of Yucatán Peninsula. (Abs.). EOS Am. Geophys. Union. vol. 50, n. ${ }^{\circ}$, pp. 313

BOWIN, C. - 1968 - Geophysical study of the Cayman Trough. Jour. Geophys. Res. vol. 73, n. ${ }^{\circ} 16$, pp. 5159.5173

CORBATO, C. - 1965 - A least squares procedure for gravity interpretation. Geophysics, vol. 30, n. ${ }^{\circ} 2$, pp. $228-233$

DEL CASTILLO, L. G. - 1969 - Nuevas técnicas de interpretación gravimétrica y magnetométrica aplicadas a la exploración minera. Mem. VII Convención Nacional de la AIMMGM, pp. $467-490$

DENGO, G. - 1968 - Estructura geológica, história tectónica y morfología de América Central. ICAITI, Guatemala

DENGO, G. - 1969 - Problems of tectonic relations between Central America and the Garibbean. Trans. Gulf Coast Assoc. Geol. Soc. vol. V, n. ${ }^{\circ}$ XIX, pp. 311-320

DONNELLY, T.W. - 1964 - Evolution of Eastern Greater Antillean Island Arc. Am. Assoc. Petroleum Geol. Bull. vol. 48, n. ${ }^{\circ}$ 5, pp. 680-696

EWING, J.; ANTOINE, J. e EWING, M. - 1960 - Geophysical measurements in the Western Caribbean Sea and in the Gulf of Mexico. Jour. Geophys. Res. vol. 65, n. ${ }^{\circ} 12$, pp. 4087-4 126 
FAHLQUIST, D.A. e DAVIES, D. K. - 1971 - Fault-block origin of the Western Cayman Ridge, Caribbean Sea. Bull. Deep-Sea Res. vol. 18, pp. 243-253

FREELAND, L. G. e DIETZ, S. R. - 1971 - Plate tectonic evolution of Caribbean-Gulf of Mexico region. Nature, vol. 232, pp. $20-23$

HESS, H.H. - 1960 - Caribbean Research Project, Progress Report. Geol. Soc. Am. Bull, vol. 71, pp. $235-240$

McBIRNEY, A. R. - 1963 - Geology of a part of the Central Guatemala Cordillera. Univ. Calif. Publ. Geol. Sci., vol. 38, pp. 177-242

MEYERHOFF, A. A.; KHUDOLEY, K. M. e HATTEN, C. W. - 1969 - Geologic significance of radiometric dates from Cuba. Am. Assoc. Petroleum Geol. Bull., vol. 53, n. ${ }^{\circ}$ 12, pp. 24942500

MOLNAR, P. e SYKES, R. L. - 1969 - Tectonics of the Caribbean and Middle America regions from focal mechanisms and seismicity. Geol. Soc. Am. Bull., vol. 80, pp. 1639-1684

MOORE, W. G. e DEL GASTILLO, G. L. - 1972 - Tectonic evolution of the Southern Gulf of Mexico. Geol. Soc. of Am., in revision

SPENGER, W. E. - 1969 - Introduction to the structure of the Earth. New York, McGraw-Hill Book Co., Inc., 597 pp

STACEY, F. D. - 1969 - Physics of the Earth. Space Science Text Series, New York, John Wiley \& Sons Inc., 324 pp

SUESS, E. - 1885 - Das Antlitz der Erde. Prag, F. Tempsky, vol. 1, 779 pp

TALWANI, M.; WORZEL, J, L. e LANDISMAN, M. - 1959 - Rapid gravity computation for two-dimensional bodies with applications to the Mendocino Submarine Fracture Zone. Jour. Geophys. Res., vol. 64, n. ${ }^{\circ}$ 10, pp. 49.59

VEDDER, J. G.; DILLON, W. P. e ROBERTS, A. E. - 1971 - USGS-IDOE LEG 2. Geotimes, vol. 16 , pp. $10-12$

VINIEGRA, F. C. - 1971 - Age and evolution of salt basins of Southeastern Mexico. Am. Assoc. Petroleum Geol. Bull., vol. 55, pp. 478-494

WORZEL, J. e SHURBET, G. L. - 1955 - Gravity interpretations from standard oceanic and continental crustal sections. Geol. Soc. Amer, Special Paper n. ${ }^{\circ} 62$, pp. 87-100 